\title{
Polypoidal choroidal vasculopathy (PCV): the 4-year review of the real-life treatment experiences
}

This article was published in the following Dove Press journal:

Clinical Ophthalmology

\section{Mansing Ratanasukon \\ Patama Bhurayanontachai \\ Pichai Jirarattanasopa}

Department of Ophthalmology, Faculty of Medicine, Prince of Songkla University, Hat Yai, Songkhla Province 90II0, Thailand
Correspondence: Mansing Ratanasukon Department of Ophthalmology, Faculty of Medicine, Prince of Songkla University, Hat Yai, Songkhla Province 90। I0, Thailand

Tel +66 74 45 I 38 I

Fax +66 74 429619

Email mratanasukon@yahoo.com
Purpose: The purpose of this article was to study the real-life treatment results of polypoidal choroidal vasculopathy (PCV).

Design: This was a retrospective study.

Methods: Patients with presumed age-related macular degeneration were reviewed, and PCV diagnosis was made using the EVEREST study criteria. Outcomes were changes in visual acuity (VA) and central retinal thickness, time between treatments, follow-up time, and number of treatments.

Results: The prevalence of PCV was $30.8 \%$. At the beginning, 195 eyes received monotherapy of anti-vascular endothelial growth factor (anti-VEGF) injections, either bevacizumab or ranibizumab, and only six eyes received the combination of anti-VEGF injection and photodynamic therapy (PDT) at the time of the first treatment. During the follow-up, some patients received "rescue or add-on" PDT when they had a poor response after anti-VEGF injections. After 4 years, the average number of injections was 8.25 and 9.15 for the anti-VEGF monotherapy and the combination groups, respectively. The average time between the first anti-VEGF injections and the first PDT was 21.4 months. The average VA in the anti-VEGF monotherapy group increased by 1.5 letters, whereas it decreased by 0.95 letters in the combination group $(P=0.48)$.

Conclusion: The review demonstrated the same visual outcomes between the combination therapy of anti-VEGF injections and rescue or add-on PDT vs monotherapy anti-VEGF injections in PCV treatment. When compared with EVEREST II and Planet studies, the "initial" or "rescue or add-on PDT" might have different effects on the final visual outcomes.

Keywords: polypoidal choroidal vasculopathy, anti-vascular endothelial growth factor, photodynamic therapy

\section{Introduction}

Polypoidal choroidal vasculopathy (PCV) usually presents with hemorrhagic or exudative maculopathy. It is characterized by polypoidal lesions with or without branching vascular network (BVN). The prevalence of PCV in Asian patients is higher than in other populations and by up to $50 \%$ in some series. ${ }^{1}$ Many studies demonstrated the treatment options of PCV, such as photodynamic therapy (PDT), intravitreal injections of anti-vascular endothelial growth factors (anti-VEGF) or steroids, and the combination treatment with various treatment outcomes. The EVEREST study ${ }^{2}$ was the first to show the benefit of the combination of PDT and anti-VEGF injection in terms of complete polyp regression. However, other studies ${ }^{3,4}$ reviewed different outcomes from PDT and the combination regimen. Recently, EVEREST II study ${ }^{5}$ has described the results of a combination of initial PDT and ranibizumab, and it showed better visual 
results than monotherapy of ranibizumab injections. In contrast, the Planet study ${ }^{6}$ demonstrated favorable outcomes from a monotherapy injection of aflibercept. Nevertheless, we still do not know the treatment outcomes from real-life experiences that could not use the same treatment regimens as described by the standard studies.

\section{Methods}

This is a real-life retrospective review of 651 patients with presumed age-related macular degeneration (AMD), who presented at Songklanagarind Hospital, Southern Thailand, from 2011 to 2014. The study was approved by the Human Research Ethics Committee, Faculty of Medicine, Prince of Songkla University, on 20 July 2017. We reviewed the patient files, fundus photographs, fluorescein angiography (FA), indocyanine green angiography (ICGA), and optical coherence tomography (OCT) by three retinal specialists. Diagnosis of PCV followed the diagnostic criteria of the EVEREST study, ${ }^{2}$ and the diagnostic agreement of at least two of the three specialists was required. The inclusion criteria were patients with clinical AMD, such as exudative, hemorrhagic, or serosanguineous maculopathy. The exclusion criteria were patients previously treated with PDT or intravitreal injections of anti-VEGF or steroids or patients with central serous chorioretinopathy, diabetic retinopathy, secondary choroidal neovascularization, and other macular conditions that were not compatible with AMD. All patients had to complete at least a 1-year follow-up. The authors collected as much data as possible, such as best-corrected visual acuity (BCVA; logMAR, using ETDRS charts), central retinal thickness (CRT) using spectral domain OCT (Spectralis; Heidelberg Engineering Inc., Heidelberg, Germany), treatments (PDT, anti-VEGF injections, and others), time between treatments, follow-up times, number of injections, and other treatments.

\section{Results}

A total of 651 patients with presumed AMD were reviewed, and 201 patients (242 eyes) were diagnosed with PCV. This showed a prevalence of $30.8 \%$. After excluding the patients with less than a 1-year follow-up, 171 patients (201 eyes) were included in the study analysis. At the beginning, 195 eyes received monotherapy of anti-VEGF injections, either bevacizumab or ranibizumab, and only six eyes received the combination of anti-VEGF injection and PDT at the time of the first treatment. The anti-VEGF injections were scheduled only when the disease showed an active condition ("prn" or "as need" injection). During follow-up,
Table I The demographic data

\begin{tabular}{|l|l|}
\hline Characteristics & $\begin{array}{l}\text { n (\%) or mean } \pm \text { SD } \\
\text { ( I 7 I patients, 20 I eyes) }\end{array}$ \\
\hline Gender & \\
\hline Male & $87(50.9)$ \\
\hline Female & $84(49.1)$ \\
\hline Mean age at PCV diagnosis, years & $68.46 \pm 9.24$ \\
\hline Laterality & \\
\hline Right eye & $80(46.8)$ \\
\hline Left eye & $61(35.7)$ \\
\hline Both eyes & $30(17.5)$ \\
\hline At baseline & \\
\hline BCVA (logMAR) & 0.6 I \pm 0.5 I \\
\hline CRT $(\mu \mathrm{m})$ & $290.39 \pm$ I I2.I5 \\
\hline
\end{tabular}

Abbreviations: PCV, polypoidal choroidal vasculopathy; BCVA, best-corrected visual acuity; $C R T$, central retinal thickness; logMAR = Logarithm of the minimum angle of resolution.

many patients received "rescue or add-on" PDT when they had a poor response or recurrence after multiple anti-VEGF injections. The poor response or recurrence was clarified or decided by the clinician preferences (mainly because of BCVA drop or new subretinal fluid from OCT).

Although this review was a retrospective study, all baseline demographic data were similar in both combination and anti-VEGF monotherapy groups, including BCVA, CRT, age, gender, and eye side (Table 1). The average follow-up time was 3.35 years, and $67 \%$ of eyes completed a 4 -year follow-up. At the beginning, 195 eyes received monotherapy injection of anti-VEGF, and only six eyes received the combination of anti-VEGF injection and "initial" PDT at the time of the first treatment. At the end of the fourth year, 79 eyes received only monotherapy injection of anti-VEGF and 55 eyes received at least one of the combination regimens during their follow-up (Table 2).

Table 2 The cumulative number of patients for each group at each year

\begin{tabular}{|l|l|}
\hline Year & $\mathbf{n}(\%)$ \\
\hline \multicolumn{2}{|l|}{ First year } \\
\hline Combination group & $34(16.9)$ \\
\hline Anti-VEGF group & $167(83.1)$ \\
\hline Second year & $47(23.7)$ \\
\hline Combination group & $151(76.3)$ \\
\hline Anti-VEGF group & $57(33.3)$ \\
\hline Third year & $114(66.7)$ \\
\hline Combination group & $55(41.0)$ \\
\hline Anti-VEGF group & $79(59.0)$ \\
\hline Fourth year &
\end{tabular}

Abbreviation: anti-VEGF, anti-vascular endothelial growth factor. 
Table 3 The number of injections at each year

\begin{tabular}{l|l|l|l}
\hline Year & Combination & Anti-VEGF & $P$-value \\
\hline First year & $3.65 \pm 2.31$ & $3.04 \pm 2.00$ & 0.116 \\
Second year & $1.85 \pm 1.99$ & $1.22 \pm 1.82$ & 0.056 \\
Third year & $1.83 \pm 2.04$ & $1.97 \pm 2.23$ & 0.672 \\
Fourth year & $2.16 \pm 2.22$ & $2.04 \pm 2.16$ & 0.743 \\
\hline
\end{tabular}

Abbreviation: anti-VEGF, anti-vascular endothelial growth factor.

After a 4-year follow-up, the average number of injections was 8.25 and 9.15 for the anti-VEGF monotherapy and the combination groups, respectively. The number of injections was similar in both groups from the first to the fourth year (Table 3 ). In the combination group, the average number of PDT was 1.26 times within the 4-year follow-up period. Interestingly, the average time between the first antiVEGF injection and the first PDT was 21.4 months. This finding indicates that most of the patients received rescue or add-on PDT in the second year of the treatment, or it meant that PDT was performed a long time after the first anti-VEGF injection.

The BCVA and CRT changes from OCT were also similar in both groups. At the end of the fourth year, the BCVA in the anti-VEGF monotherapy group had increased by an average of 1.5 letters $(-0.03 \log$ MAR), whereas it had decreased by an average of 0.95 letters $(+0.019 \log \mathrm{MAR})$ in the combination group ( $P=0.48$; Figure 1$)$. Nevertheless, the OCT or CRT changes showed interesting results, demonstrating the benefit of the combination treatment, especially in the third year (Figure 2). For the categories of visual acuity (VA) changes, gain or loss, the data showed that the proportion of

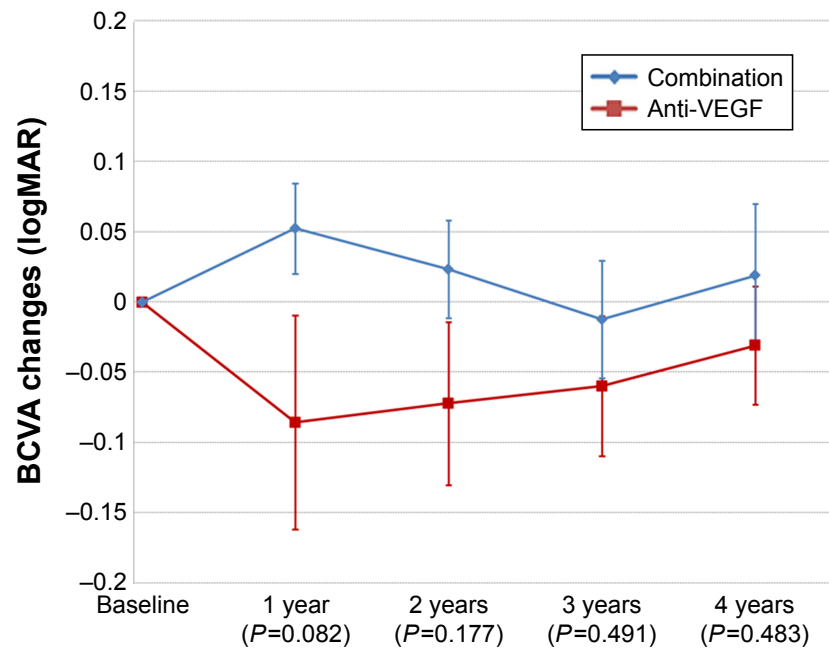

Figure I The BCVA changes from the baseline for the combination and anti-VEGF groups at each year.

Abbreviations: BCVA, best-corrected visual acuity; anti-VEGF, anti-vascular endothelial growth factor.

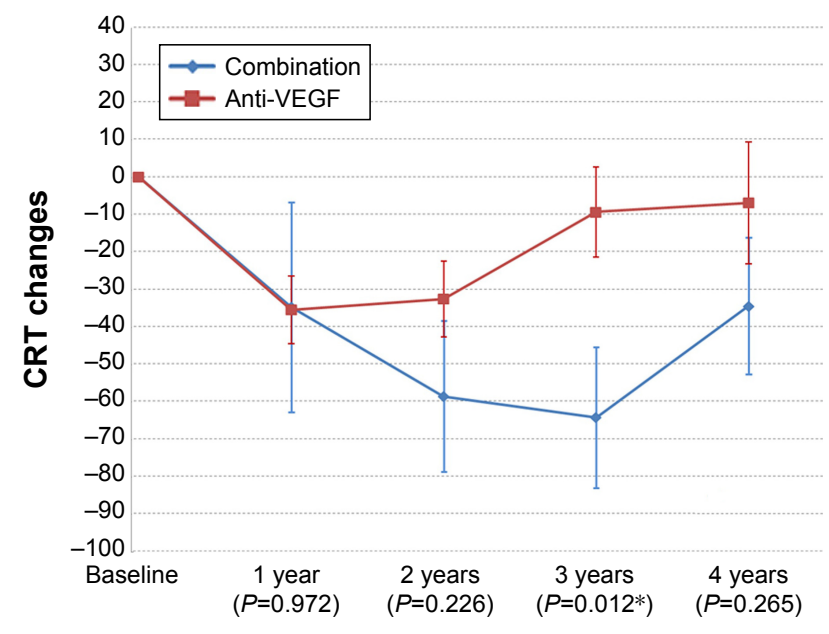

Figure 2 The CRT changes from the baseline for the combination and anti-VEGF groups at each year.

Note: *P-value with statistical significance.

Abbreviations: CRT, central retinal thickness; anti-VEGF, anti-vascular endothelial growth factor.

patients with VA gain or loss were also similar in both groups for most of the categories of VA changes (Table 4).

\section{Discussion}

The review demonstrated a prevalence of PCV in Thai patients of $30.8 \%$, which is comparable with other Asian populations. This finding means that about one-third of Thai patients with presumed AMD would have PCV. Our prevalence was lower than the previous Thai PCV study ${ }^{1}$ because we used the EVEREST-diagnostic criteria strictly, and all diagnoses were made by at least two of three experts in agreement. However, this was not a real "prevalence" because the review was retrospective and not an exact population-based study. In our real-life practice, most patients received antiVEGF injection as the first line of treatment, followed by rescue or add-on PDT only when they had a poor response or recurrence from multiple injections. Our rescue or add-on was different from "rescue" PDT of the Planet study ${ }^{6}$ in terms of lacking strict treatment protocols and depending on only clinicians' preferences or decisions. Therefore, our results could not be directly compared with the results from the Planet study. Moreover, our combination regimen was also different from that of the EVEREST II study that used PDT as the initial treatment with anti-VEGF injection.

From the review, our real-life treatment regimens could be divided into two main groups: the first was the monotherapy prn anti-VEGF injection and the second was a combination of prn anti-VEGF injection and rescue or add-on PDT. The VA results were similar in both groups from the first to fourth year. All categories of VA changes, either VA gain or loss, were also similar. The average VA changes compared to 
Table 4 The number of patients for categories of VA changes at each year

\begin{tabular}{|c|c|c|c|}
\hline BCVA & $\begin{array}{l}\text { Combination } \\
(n, \%)\end{array}$ & $\begin{array}{l}\text { Anti-VEGF } \\
(n, \%)\end{array}$ & $P$-value \\
\hline \multicolumn{4}{|l|}{ First year } \\
\hline BCVA gain > I line & $7(20.6)$ & $60(35.9)$ & 0.110 \\
\hline BCVA gain $>2$ lines & $6(17.6)$ & $47(28.1)$ & 0.286 \\
\hline BCVA gain $>3$ lines & $5(14.7)$ & $29(17.4)$ & 0.807 \\
\hline BCVA loss $\leq$ I line & $25(73.5)$ & $143(85.6)$ & 0.124 \\
\hline BCVA loss $\leq 2$ lines & $26(76.5)$ & I5I (90.4) & $0.038^{*}$ \\
\hline BCVA loss $\leq 3$ lines & $27(79.4)$ & $152(91.0)$ & 0.067 \\
\hline \multicolumn{4}{|l|}{ Second year } \\
\hline BCVA gain > I line & I3 (27.7) & $59(39.1)$ & 0.169 \\
\hline BCVA gain $>2$ lines & $10(21.3)$ & $40(26.5)$ & 0.566 \\
\hline BCVA gain $>3$ lines & $6(12.8)$ & $11(13.9)$ & 1.000 \\
\hline BCVA loss $\leq$ I line & $34(72.3)$ & $122(80.8)$ & 0.225 \\
\hline BCVA loss $\leq 2$ lines & $38(80.9)$ & I33 (88.1) & 0.227 \\
\hline BCVA loss $\leq 3$ lines & $38(80.9)$ & 137 (90.7) & 0.073 \\
\hline \multicolumn{4}{|l|}{ Third year } \\
\hline BCVA gain > I line & $21(36.8)$ & $43(37.7)$ & 1.000 \\
\hline BCVA gain $>2$ lines & $13(22.8)$ & $30(26.3)$ & 0.710 \\
\hline BCVA gain $>3$ lines & $7(12.3)$ & $17(14.9)$ & 0.816 \\
\hline BCVA loss $\leq \mathrm{I}$ line & $46(80.7)$ & $85(74.6)$ & 0.445 \\
\hline BCVA loss $\leq 2$ lines & $48(84.2)$ & $95(83.3)$ & 1.000 \\
\hline BCVA loss $\leq 3$ lines & $49(86.0)$ & $100(87.7)$ & 0.810 \\
\hline \multicolumn{4}{|l|}{ Fourth year } \\
\hline BCVA gain > I line & $18(32.7)$ & $25(31.6)$ & 1.000 \\
\hline BCVA gain $>2$ lines & $10(18.2)$ & $20(25.3)$ & 0.402 \\
\hline BCVA gain $>3$ lines & $5(9.1)$ & II (13.9) & 0.433 \\
\hline BCVA loss $\leq$ I line & $43(78.2)$ & $53(67.1)$ & 0.178 \\
\hline BCVA loss $\leq 2$ lines & $47(85.5)$ & $62(78.5)$ & 0.371 \\
\hline BCVA loss $\leq 3$ lines & $48(87.3)$ & $68(86.1)$ & 1.000 \\
\hline
\end{tabular}

Abbreviations: VA, visual activity; BCVA, best-corrected visual acuity; anti-VEGF, anti-vascular endothelial growth factor.

baseline were increases of 1.5 letters and decreases of 0.95 letters in the monotherapy anti-VEGF injections and the combination groups, respectively. In general, VA was lower than the results from standard studies ${ }^{2-6}$ because our review was conducted in real-life practice and we did not use the injection protocol strictly, leading to lower visual outcomes. Moreover, when compared with the EVEREST II study, our results did not demonstrate the BCVA benefit from the combination regimen. These findings might come from two possible reasons. First, our PDT was done in nonresponsive cases that usually had poorer vision; thus, the additional effect of PDT might not overcome the better visual results from the monotherapy group. Second, the rescue or add-on PDT itself might have been less effective than the initial combination regimen in terms of visual outcomes. Our review used PDT at the average time of 21.4 months, which was a long time after the first injection. Hence, this might give different results from the combination study of initial or "early"
PDT as shown by the EVEREST II and Fujisan studies. ${ }^{4,5}$ We postulated that the multiple anti-VEGF injections might change some histological structures of the polyps or BVN and some treatment responses from PDT. This hypothesis also explains the finding from the EVEREST II study that showed the benefit of PDT because it used PDT only as the initial treatment with anti-VEGF injection. Therefore, from all the recent data, if we consider the combination regimen for PCV treatment, we might better choose initial rather than rescue or add-on PDT.

The CRT studies from the review also showed similar results in both treatment groups, except in the third year that demonstrated some benefit of the combination regimen. These findings might be the effect of PDT in the second to third year because most of our patients received rescue or add-on PDT at the average time of 21.4 months. The treatment in the second year would make the CRT thinner in the third year, although it did not affect the visual outcomes as described.

The limitation of this study was its retrospective design. The design could not control many treatment protocols or schedules. The reinjection criteria were planned only by the experts' opinions and made our regimens and results different from the standard studies. However, the results could give us the answers about real-life practices and the treatment results of PCV, especially in Thailand. The recurrent rates were also difficult to determine because there were many reinjections for different reasons during this long-term follow-up review. Moreover, because some of the cases in our review received both bevacizumab and ranibizumab, we could not evaluate the different effects of both drugs on the visual outcomes. This limitation might be a major confounding factor of this retrospective review.

\section{Conclusion}

Our real-life review demonstrated the same visual treatment outcomes between the combination therapy of anti-VEGF injections and "add-on" PDT vs monotherapy prn anti-VEGF injections in PCV treatment. When compared with the EVEREST II and the Planet studies, the "initial" or "rescue or add-on PDT" might have different effects on the final visual outcomes.

\section{Ethics statement}

The study was approved by the Human Research Ethics Committee, Faculty of Medicine, Prince of Songkla University, on 20 July 2017 (EC 60-128-02-1) Chairman of the Human Research Ethics Committee; Associate Professor Boonsin 
Tangtrakulwanich. All patients signed informed consents for all treatments. The Human Research Ethics Committee, Faculty of Medicine, Prince of Songkla University, does not require the patient informed consent for their medical record reviews (because some patients passed away or are lost to follow-up), but all retrospective proposals must be approved before the reviews. All patient data were confidentially protected by hospital personnel passwords (all patient medical data are electronic files). Therefore, all data reviews must be approved by the committee.

\section{Author contributions}

The corresponding author MR made substantial contribution to conception and design, analysis and interpretation of data, drafting the article, and final approval of the version to be published. PB and PJ carried out acquisition, analysis and interpretation of data. All authors contributed toward data analysis, drafting and critically revising the paper, gave final approval of the version to be published, and agree to be accountable for all aspects of the work.

\section{Disclosure}

The authors report no conflicts of interest in this work.

\section{References}

1. Bhoomibunchoo C, Yospaiboon Y, Thoongsuwan S, et al. Idiopathic polypoidal choroidal vasculopathy in Thai patients with clinical and angiographic choroidal neovascularization. Clin Ophthalmol. 2017;11(11): 317-322.

2. Koh A, Lee WK, Chen LJ, et al. EVEREST study: efficacy and safety of verteporfin photodynamic therapy in combination with ranibizumab or alone versus ranibizumab monotherapy in patients with symptomatic macular polypoidal choroidal vasculopathy. Retina. 2012;32(8): 1453-1464.

3. Oishi A, Kojima H, Mandai M, et al. Comparison of the effect of ranibizumab and verteporfin for polypoidal choroidal vasculopathy: 12-month LAPTOP study results. Am J Ophthalmol. 2013;156(4):644-651.

4. Gomi F, Oshima Y, Mori R, et al. Initial versus delayed photodynamic therapy in combination with ranibizumab for treatment of polypoidal choroidal vasculopathy: The Fujisan study. Retina. 2015;35(8):1569-1576.

5. Koh A, Lai TYY, Takahashi K, et al. Efficacy and safety of ranibizumab with or without verteporfin photodynamic therapy for polypoidal choroidal vasculopathy: a randomized clinical trial. JAMA Ophthalmol. 2017;135(11):1206-1213.

6. Lee WK, Iida T, Ogura Y, et al. Efficacy and Safety of Intravitreal Aflibercept for Polypoidal Choroidal Vasculopathy in the PLANET Study: A Randomized Clinical Trial. JAMA Ophthalmol. 2018;136(7):786-793.
Clinical Ophthalmology

\section{Publish your work in this journal}

Clinical Ophthalmology is an international, peer-reviewed journal covering all subspecialties within ophthalmology. Key topics include: Optometry; Visual science; Pharmacology and drug therapy in eye diseases; Basic Sciences; Primary and Secondary eye care; Patien Safety and Quality of Care Improvements. This journal is indexed on

\section{Dovepress}

PubMed Central and CAS, and is the official journal of The Society of Clinical Ophthalmology (SCO). The manuscript management system is completely online and includes a very quick and fair peer-review system, which is all easy to use. Visit http://www.dovepress.com/ testimonials.php to read real quotes from published authors. 